\title{
Prevalence of night-time dyspnoea in COPD and its implications for prognosis
}

\author{
Peter Lange ${ }^{1,2,3,4}$, Jacob Louis Marott ${ }^{3}$, Jørgen Vestbo $0^{5,6}$ and \\ Børge Grønne Nordestgaard $3,4,7,8$
}

\begin{abstract}
Affiliations: 'Section of Social Medicine, Dept of Public Health, University of Copenhagen, Copenhagen, ${ }^{2}$ Respiratory Section, Hvidovre Hospital, Copenhagen University Hospital, Copenhagen, ${ }^{3}$ The Copenhagen City Heart Study, Frederiksberg Hospital, Copenhagen University Hospital, Copenhagen, ${ }^{4}$ The Copenhagen General Population Study, Herlev Hospital, Copenhagen University Hospital, Herlev, ${ }^{5}$ Dept of Respiratory Medicine, Odense University Hospital, University of Southern Denmark, Odense, ${ }^{7}$ Dept of Clinical Biochemistry, Herlev Hospital, Copenhagen University Hospital, Herlev, and ${ }^{8}$ Faculty of Health and Medical Sciences, University of Copenhagen, Copenhagen, Denmark. ${ }^{6}$ Respiratory and Allergy Research Group, Manchester Academic Health Science Centre, University of Manchester, Manchester, UK.
\end{abstract}

Correspondence: P. Lange, Section of Social Medicine, Dept of Public Health, University of Copenhagen, P.O.B. 2099, Øster Farimagsgade 5, DK-1014 Copenhagen K, Denmark. E-mail: peter.langedsund.ku.dk

ABSTRACT The information on night-time symptoms in chronic obstructive pulmonary disease (COPD) is sparse.

We investigated the prevalence of night-time dyspnoea in 6616 individuals with COPD recruited from the general population in the Copenhagen area, Denmark, and described characteristics and prognosis of subjects with this symptom.

The prevalence of night-time dyspnoea was 4.3\%: 2.1\% in Global Initiative for Chronic Obstructive Lung Disease (GOLD) group A, $12.9 \%$ in GOLD B, 2.6\% in GOLD C and 16.3\% in GOLD D. Compared with individuals without night-time dyspnoea, those with night time dyspnoea had lower forced expiratory volume in $1 \mathrm{~s}$, higher daytime dyspnoea scores (modified Medical Research Council scale) and more wheezing, more often had chronic mucus hypersecretion, ischaemic heart disease and atrial fibrillation, and more often reported stress, nervousness and tiredness. After adjustment for age and sex, the presence of night-time dyspnoea was associated with future COPD exacerbations (hazard ratio (HR) 2.3, 95\% CI 1.7-3.0), hospital admissions due to COPD (HR 3.2, 95\% CI 2.3-4.4) and mortality (HR 1.7, 95\% CI 1.2-2.3).

Prevalence of night-time dyspnoea in COPD increases with disease severity according to both spirometric and clinical GOLD classification, and is associated with presence of daytime respiratory symptoms and cardiac comorbidities. Night-time dyspnoea is a significant predictor of poor prognosis in individuals with COPD.

@ERSpublications

Night-time dyspnoea in COPD is significantly related to disease severity and to poor prognosis http://ow.ly/sDRIf

For editorial comments see page 1560 .

Received: Nov 122013 | Accepted after revision: Dec 312013 | First published online: Jan 312014

Support statement: The study was funded by the Capital Region of Copenhagen, Danish Heart Foundation, Danish Lung Foundation, Velux Foundation, Herlev Hospital and Almirall. The funding sources had no role in the design or conduct of the study, in the collection, management, analysis or interpretation of the data, or in the preparation, review, or approval of the manuscript

Conflict of interest: Disclosures can be found alongside the online version of this article at www.erj.ersjournals.com

Copyright @ERS 2014 


\section{Introduction}

Until recently, there has not been much focus on night-time symptoms in chronic obstructive pulmonary disease (COPD). This is in contrast to asthma, where night-time respiratory symptoms have been recognised for many years and are included in the clinical assessment of disease control [1]. Yet, in connection with the development of the COPD assessment test (CAT), sleep disturbance has emerged as an important factor related to quality of life in COPD [2]. As the inclusion of the questions in CAT was mainly a patient-driven process, unlike earlier expert-composed COPD questionnaires, it is possible that the prevalence of night-time symptoms has been underreported in the past due to a lack of focus on these symptoms from doctors treating COPD patients. In addition, an expert panel recently concluded that the prevalence of night-time symptoms in COPD seems to be high and that there is a need for more research on this topic [3]. By reviewing the relatively meagre literature on night-time symptoms in COPD, the expert panel concluded that these symptoms may be caused by a number of factors including COPD severity, obesity and comorbidity including sleep disorders. In addition, the consequences of night-time disturbances for the COPD patient, in addition to a reduced quality of life, could be a worse prognosis including frequent exacerbations and poorer survival [3].

The present study was undertaken to investigate the prevalence of night-time dyspnoea among individuals with COPD recruited from the general population. In addition to investigating the predictors of presence of night-time dyspnoea, we also aimed to investigate the association between this symptom and clinical prognosis including future exacerbations, hospital admissions due to COPD and mortality.

\section{Material and methods \\ Study subjects}

We pooled data from two similar but independent studies: the fourth examination of the Copenhagen City Heart Study (CCHS) in 2001-2003 and the examination of the Copenhagen General Population Study (CGPS) in 2003-2008. These studies were approved by institutional review boards and Danish ethics committees (KF100.2039/91 and H-KF01-144/01), and were conducted according to the Declaration of Helsinki. Written informed consent was obtained from all participants.

\section{Copenhagen City Heart Study}

The CCHS is a prospective epidemiological study initiated in 1976-1978. A sample of 19698 subjects aged 20-100 years was selected at random from the national Danish Civil Registration System, after age stratification in 5-year age groups, from residents of central Copenhagen, Denmark, and 14223 subjects participated in the initial survey at Copenhagen University Hospital [4]. They were all re-invited to participate in later surveys along with additional subjects in the youngest age groups. A total of 6237 attended the fourth survey in 2001-2003 (response rate 50\%), which is the survey we used as the baseline in the present analyses [5].

\section{Copenhagen General Population Study}

The CGPS is a prospective epidemiological study that aims to recruit $>100000$ subjects representative of the general population of the suburbs of Copenhagen, and collect genotypic and phenotypic data of relevance to a wide range of health-related problems. It is designed almost identically to the CCHS. Recruitment began in 2003 and is still ongoing (response rate 45\%) [6].

\section{Study parameters}

In both studies, participants filled in an extensive questionnaire concerning lifestyle and health topics, including medication use at home. A physical examination was performed, where basic variables such as weight and height were measured. Body mass index (BMI) was calculated as weight divided by height squared. Physical activity, respiratory symptoms (chronic mucus hypersecretion and wheezing), comorbidities (heart disease and diabetes) and general symptoms (feeling of stress, tiredness and nervousness) were reported in the questionnaire.

A Vitalograph spirometer (Maids Moreton, UK) was used in the CCHS and in the first 14624 participants in the CGPS. An EasyOne Diagnostic Spirometer (ndd Medizintechnik, Zurich, Switzerland) was used in the remaining participants in the CGPS. Spirometry was performed in the standing position without the use of a nose clip. Three sets of forced expiratory volume in $1 \mathrm{~s}$ (FEV1) and forced vital capacity (FVC) values were obtained. As a criterion for correct performance of the procedure, at least two measurements differing by $<5 \%$ had to be produced together with the correct visual appearance of the volume-time tracings with the Vitalograph and flow-volume tracings with the EasyOne spirometer. We used the highest obtained values for every single participant of both FEV1 and of FVC. Only pre-bronchodilatory measurements were available. 


\section{Study design}

Individuals with COPD were identified according to international COPD guidelines, based on FEV1/FVC $<0.7$. In accordance with the Global Initiative for Chronic Obstructive Lung Disease (GOLD) document, we excluded individuals with self-reported asthma [7].

In the present analyses, we only included individuals $\geqslant 40$ years of age. Among 5919 participants of the CCHS and 55731 participants of the CGPS, we identified 6616 individuals fulfilling the aforementioned COPD criteria who also had information on night-time symptoms. We also performed sensitivity analyses where we only included 4855 individuals who, in addition to $\mathrm{FEV} 1 / \mathrm{FVC}<0.7$, also had FEV1/FVC below the lower limit of normal (LLN) according to recently published reference values for the CCHS and CGPS $(\mathrm{n}=4855)[8]$.

In all analyses, the presence of night-time dyspnoea was defined in the participants who answered positively to the question: do you sometimes wake up in the night because of breathlessness/difficulties with breathing? We have no information on other symptoms like coughing, wheezing or mucus hypersecretion during the night.

In the analyses, we included various demographic and clinical variables describing the participants with COPD on the basis of the information obtained in the questionnaire at the time of the examination. We used both the GOLD 2007 1-4 classification based on FEV1 as a percentage of the predicted value and the GOLD 2011 classification with groups A-D according to a combination of $\mathrm{FEV}_{1} \%$ pred, self-reported severity of dyspnoea (modified Medical Research Council (mMRC) scale) and frequency of COPD exacerbations in the year prior to the baseline examination [7]. To define exacerbations both in the year before the baseline examination (in order to classify participants into GOLD groups A-D) and during the follow-up (in order to register end-points for the analyses), we used information regarding hospital admissions due to COPD and the use of oral corticosteroids and antibiotics used to treat the exacerbations. The methodology for obtaining these data has previously been described in detail $[9,10]$.

\section{Clinical outcomes}

After the examination date, the participants were followed by means of the nationwide registries for up to 8.9 years with an average of 4.3 years. The following clinical outcomes were defined: COPD exacerbation (defined based on the medication used), admission to hospital due to COPD and all-cause mortality. This was done using the unique personal civil registration number assigned to all Danish inhabitants by linking our surveys to three national registries: the Danish Registry of Medicinal Product Statistics, which contains information on all prescriptions dispensed in all Danish pharmacies [11]; the Danish National Patient Registry covering all hospital contacts in Denmark; and the Danish Central Person Register, which contains information on all deaths in Denmark. We identified the relevant medications using the Anatomic Therapeutic Chemical) code. From the Danish National Patient Registry, we identified hospital admissions with a discharge diagnosis of COPD (International Classification of Diseases, 10th edn: J41-J44). An exacerbation of COPD was defined as a short course of treatment with prednisolone alone or in combination with an antibiotic, or an acute admission to hospital because of COPD.

\section{Statistical analysis}

All analyses were performed with R version 3.0.1 (R Foundation for Statistical Computing, Vienna, Austria) [12]. For demographics, ANOVA was used for continuous variables and Fisher's exact tests were used for categorical variables.

Multivariate logistic regression analysis was applied to investigate which participants' characteristics were cross-sectionally associated with self-reported night-time dyspnoea at the baseline examination. In addition to age and sex, considered as basic variables, we included variables that differed significantly between the individuals with and without night-time dyspnoea as covariates. These included GOLD A-D grouping, BMI, physical activity, respiratory symptoms (chronic mucus hypersecretion and wheezing), comorbidities (heart disease and diabetes) and general symptoms (feeling of stress, tiredness and nervousness). As the frequency of exacerbations, daytime breathlessness and FEV1 \% pred are included in the GOLD A-D classification, these variables were not included in the model as separate covariates. p-values $<0.05$ were considered significant.

In the longitudinal analyses, the log-rank test was used to compare the difference in exacerbations, hospital admissions with COPD, and all-cause mortality between individuals with and without night-time dyspnoea. To estimate the relative risk of the clinical events for these two subgroups, we used a Cox regression model with age as the underlying time scale. In so-called univariate models, only sex was included in addition to the presence of night-time dyspnoea, which was the variable of main interest. In the two multivariable models, we also included the spirometry-based GOLD 1-4 and the clinical GOLD A-D groups as covariates. 
TABLE 1 Prevalence of night time dyspnoea according to different characteristics describing individuals with chronic obstructive pulmonary disease

Prevalence of night-time dyspnoea

p-value

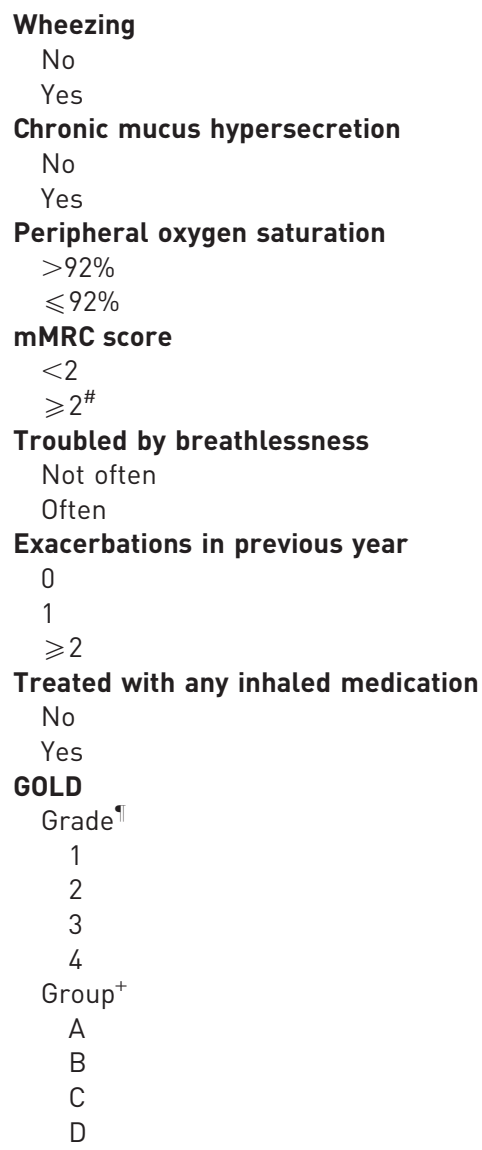

$2.1(98 / 4691)$

$9.8(180 / 1835)$

$2.7(145 / 5288)$

$10.5(135 / 1288)$

$4.1(265 / 6438)$

$10.0(14 / 140)$

$2.1(115 / 5392)$

$13.7(168 / 1224)$

$2.3(139 / 5977)$

$23.7(142 / 600)$

$4.0(257 / 6373)$

$7.0(8 / 114)$

$14.0(18 / 129)$

$3.6(212 / 5956)$

$10.8(71 / 660)$

$3.0(99 / 3301)$

$4.9(139 / 2845)$

$8.9(38 / 425)$

$15.6(7 / 45)$

$2.1(108 / 5121)$

$12.9(120 / 930)$

$2.6(7 / 271)$

$16.3(48 / 294)$
$<0.001$

0.002
Data are presented as \% (number of individuals with night-time dyspnoea/number of individuals with the characteristic of interest), unless otherwise stated. mMRC: modified Medical Research Council; GOLD: Global Initiative from Chronic Obstructive Lung Disease. " : participant walks more slowly than people of the same age on the level due to breathlessness; " ${ }^{\circ}$ : spirometric classification according to forced expiratory volume in $1 \mathrm{~s}$ (FEV1) as a percentage of the predicted value; ${ }^{+}$: clinical classification according to FEV $1 \%$ pred, symptom burden and frequency of exacerbations.

\section{Results}

From among 61650 individuals participating in the two studies, a total of 6616 individuals fulfilled our COPD inclusion criteria based on age, FEV1/FVC $<0.7$ and absence of asthma, and had the necessary information on night-time dyspnoea.

The overall prevalence of night-time dyspnoea in the COPD study population was $4.3 \%$. The prevalence was the same in males and females, and smokers and nonsmokers, and did not differ in the individuals below and above 60 years of age. We observed a U-shaped distribution with regard to BMI, with the most undernourished $\left(\mathrm{BMI}<18.5 \mathrm{~kg} \cdot \mathrm{m}^{-2}\right)$ and most overweight subjects $\left(\mathrm{BMI}>30 \mathrm{~kg} \cdot \mathrm{m}^{-2}\right.$ ) reporting highest prevalence of night-time dyspnoea of $6.9 \%$ and $6.2 \%$, respectively, whereas the prevalence in individuals with BMI between 18.5 and $25 \mathrm{~kg} \cdot \mathrm{m}^{-2}$ was $3.3 \%(\mathrm{p}<0.001)$.

Night-time dyspnoea was significantly more prevalent in individuals, who also reported daytime respiratory symptoms and had day time peripheral oxygen saturation $<92 \%$ (table 1 ). This was most pronounced in individuals who reported being often troubled by breathlessness and those with an mMRC score $\geqslant 2$. Individuals with frequent exacerbations and those treated with inhaled medications reported more nighttime dyspnoea (table 1). The prevalence of night-time dyspnoea according to the spirometry-based GOLD 
1-4 stratification and the multidimensional clinical GOLD A-D grouping is also shown in table 1. As expected, the highest prevalences were observed in the most severe groups, GOLD 4 and D, where they were $15.6 \%$ and $16.3 \%$, respectively. The number of participants diagnosed with sleep apnoea was small: 22 in GOLD A, six in GOLD B, two in GOLD C and none in GOLD D.

Individuals with night-time dyspnoea had, on average, a lower FEV1 \% pred, were more often troubled by breathlessness and a significantly higher percentage of them had an mMRC score $\geqslant 2$ (table 2). They also had a significantly higher prevalence of wheezing, chronic mucus hypersecretion, and a higher frequency of exacerbations and episodes of acute bronchitis in the past. The frequency of current smoking did not differ significantly between the two groups, but those with night-time dyspnoea had more often been exposed to dust and fumes at work. They had also a higher frequency of comorbidities and were likely to feel nervous or tired (table 2).

The results of the multiple logistic regression analysis describing the odds ratio of having night-time dyspnoea compared with no night-time dyspnoea are shown in table 3. Table 3 also shows the same analysis in individuals with $\mathrm{FEV}$ / FVC both $<0.7$ and $<$ LLN. It is important to notice that the GOLD A-D classification includes a dimension describing daytime dyspnoea and that, in contrast to GOLD groups A and $\mathrm{C}$, individuals in groups $\mathrm{B}$ and $\mathrm{D}$ have a mMRC score $\geqslant 2$, indicating breathlessness while walking on the level with people of the same age. The model shows that the latter groups also have a higher risk of experiencing dyspnoea during the night (table 3). In addition, daytime pulmonary symptoms like wheezing and chronic mucus hypersecretion, and presence of ischaemic heart disease, atrial fibrillation, stress/ nervousness and tiredness were significantly associated with night-time dyspnoea, whereas the association with diabetes mellitus was not significant. The analysis of the participants with FEV1/FVC both $<0.7$ and $<$ LLN gave similar results (table 3 ).

During follow-up, 687 individuals experienced COPD exacerbations, 359 were admitted to hospital due to COPD and 591 died. Figure 1 shows the Kaplan-Meier curves of individuals with and without night-time dyspnoea for these three outcomes. According to the log-rank test, individuals with night-time dyspnoea had a significantly poorer prognosis (fig. 1).

Table 4 shows the results of the analyses describing night-time dyspnoea as a predictor of subsequent exacerbations, admissions because of COPD and all-cause mortality. In the Cox model with adjustment for

TABLE 2 Characteristics of individuals with chronic obstructive pulmonary disease without and with night-time dyspnoea

\begin{tabular}{|c|c|c|c|}
\hline & No night-time dyspnoea & Night-time dyspnoea & p-value \\
\hline Age years & $66.5 \pm 11.5$ & $66.2 \pm 11.9$ & 0.760 \\
\hline Males & 47.3 & 50.2 & 0.362 \\
\hline FEV $1 \%$ pred & $79.7 \pm 19.3$ & $71.4 \pm 20.7$ & $<0.001$ \\
\hline BMI $\mathrm{kg} \cdot \mathrm{m}^{-2}$ & $25.5 \pm 4.2$ & $26.4 \pm 4.5$ & $<0.001$ \\
\hline mMRC score $\geqslant 2^{\#}$ & 16.7 & 59.4 & $<0.001$ \\
\hline Often troubled by breathlessness & 7.3 & 50.5 & $<0.001$ \\
\hline Peripheral oxygen saturation $\leqslant 92 \%$ & 2.0 & 5.0 & 0.002 \\
\hline Wheezing & 26.5 & 64.7 & $<0.001$ \\
\hline Chronic mucus hypersecretion & 18.3 & 48.2 & $<0.001$ \\
\hline$>5$ episodes of acute bronchitis in previous 10 years & 3.2 & 18.7 & $<0.001$ \\
\hline$\geqslant 2$ exacerbations in previous year & 1.8 & 6.4 & $<0.001$ \\
\hline Current smokers & 40.2 & 43.6 & 0.284 \\
\hline Sedentary & 9.4 & 14.9 & 0.004 \\
\hline Exposed to dust/fumes at work & 15.2 & 24.5 & $<0.001$ \\
\hline Treated with any inhaled medication & 9.3 & 25.1 & $<0.001$ \\
\hline On cardiac medication & 9.6 & 22.8 & $<0.001$ \\
\hline Previous admission for IHD & 9.0 & 22.6 & $<0.001$ \\
\hline Diabetes & 4.4 & 8.5 & 0.003 \\
\hline Atrial fibrillation & 4.5 & 9.9 & $<0.001$ \\
\hline Feeling nervous/stressed & 21.4 & 43.7 & $<0.001$ \\
\hline Feeling tired & 41.7 & 76.6 & $<0.001$ \\
\hline
\end{tabular}

Data are presented as mean \pm SD or $\%$, unless otherwise stated. FEV 1 : forced expiratory volume in $1 \mathrm{~s}$; BMI: body mass index; mMRC: modified Medical Research Council; IHD: ischaemic heart disease. " : participant walks more slowly than people of the same age on the level due to breathlessness. 
TABLE 3 Multiple logistic regression model predicting night time dyspnoea with sex, age, body mass index (BMI), physical activity, Global Initiative for Chronic Obstructive Lung Disease (GOLD) groups A-D, chronic mucus hypersecretion, wheezing, ischemic heart disease, atrial fibrillation and diabetes mellitus as predictors

Variable
Individuals with FEV1/FVC $<0.7$
Individuals with $\mathrm{FEV}_{1} / \mathrm{FVC}<0.7$ and $<\mathrm{LLN}$

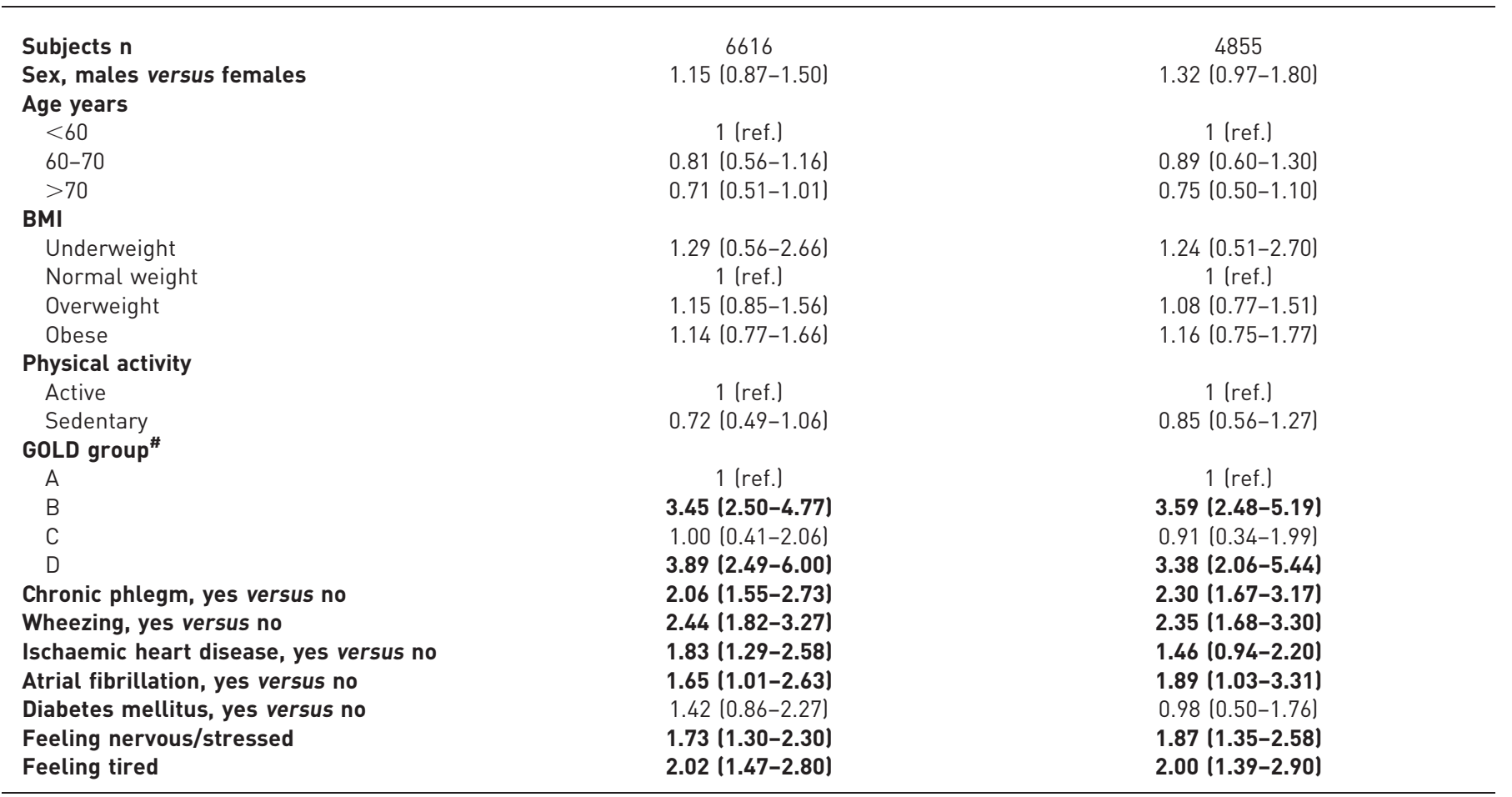

Data are presented as odds ratio (95\% confidence interval), unless otherwise stated. The odds ratio is considered statistically significant if the confidence interval does not include 1 (bold). FEV1: forced expiratory volume in $1 \mathrm{~s}$; FVC: forced vital capacity; LLN: lower limit of normal. \#: clinical classification according to FEV 1 as a percentage of the predicted value, symptom burden and frequency of exacerbations. sex and age (the latter as underlying time scale), night-time dyspnoea was significantly associated with higher risk for all the investigated end-points: the presence of night-time dyspnoea was associated with future COPD exacerbations. After additional inclusion of the spirometric GOLD 1-4 staging, night-time dyspnoea was significantly related to COPD exacerbations and COPD admissions only, whereas the relationship between night-time dyspnoea and mortality was of borderline significance $(p=0.07)$. When we included clinical GOLD A-D staging in the Cox model, night-time dyspnoea was significantly related to COPD exacerbations only with a hazard ratio of 1.4 , but not to admissions and mortality (table 4). In our supplementary analyses comprising individuals with FEV1/FVC both $<0.7$ and $<$ LLN, night-time dyspnoea was even more strongly related to the investigated end-points. In the multivariable model with age, sex and GOLD $1-4$ as covariates, the hazard ratio was 2.15 (95\% CI 1.60-2.88) for exacerbations, 2.13 (95\% CI 1.51-3.00) for COPD admissions and 1.65 (95\% CI 1.15-2.36) for total mortality.

\section{Discussion}

The present study shows that night-time dyspnoea in COPD is strongly related to disease severity, and is associated with lower $\mathrm{FEV}_{1}$, higher day time dyspnoea score (mMRC), higher prevalence of bronchitis and wheezing, and more previous episodes of acute bronchitis and exacerbations. In addition, the presence of ischaemic heart disease, atrial fibrillation, diabetes, stress, nervousness and tiredness, and either very low or very high BMI were associated with night-time dyspnoea. Our findings agree with the few previous reports available, where similar patient characteristics were found to be associated with prevalence of night-time disturbances in COPD. In the study by OMACHI et al. [13], sleep disturbance was investigated in 98 COPD patients with a mean FEV1 of $\sim 50 \%$ pred. Sleep disturbance was significantly related to cough and daytime dyspnoea, but surprisingly, not to FEV1 [13]. In the study by PRICE et al. [14], primary care physicians and respiratory specialists in five European countries recruited consecutive patients with COPD and asked questions on both day- and night-time symptoms. A surprisingly high prevalence of night-time symptoms 

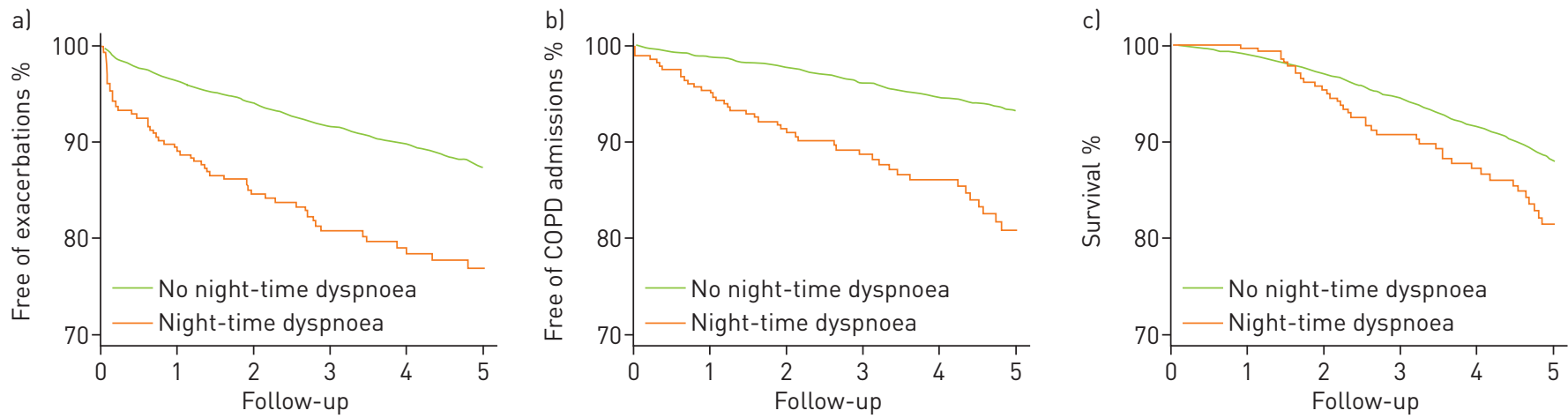

FIGURE 1 a) Exacerbations $(\mathrm{p}<0.001)$, b) hospital admissions due to chronic obstructive pulmonary disease $(\mathrm{COPD})(\mathrm{p}<0.001)$ and $\mathrm{c})$ survival $(\mathrm{p}=0.004)$ shown as Kaplan-Meier curves according to absence or presence of night-time dyspnoea. Significance values are based on the log-rank test.

of $67 \%$ was recorded. Patients with night-time symptoms were more likely to have more severe daytime breathlessness, more exacerbations and receive more inhaled medications for their COPD [14]. As in our study, there was no clear association between sex and smoking status and night-time symptoms, but individuals reporting night-time symptoms were older than those with daytime symptoms only [14].

The overall prevalence of night-time dyspnoea in our sample was relatively low (4.3\%). This low prevalence is most likely related to our recruitment method, using a general population approach and resulting in $77 \%$ of the individuals with COPD belonging to the mild GOLD A category. In the more symptomatic categories, GOLD B and GOLD D, the prevalence of awakening due to night time dyspnoea was $12.9 \%$ and $16.3 \%$, respectively.

It is noteworthy that night-time dyspnoea was more prevalent in GOLD B than in GOLD C even though FEV1 is lower in the latter group. This reflects a connection between day- and night-time symptoms, although we cannot be certain that these symptoms are actually caused by COPD. Since neither the CCHS nor the CGPS were specifically designed to investigate night-time symptoms in COPD, sleep monitoring was not conducted and, thus, we can only speculate on possible mechanisms behind the self-reported nighttime breathlessness and trouble with breathing in our COPD subjects. It is actually possible that these awakenings were caused by respiratory symptoms other than dyspnoea, but the patients perceived them as problems with breathing.

Although the few clinical studies available report up to $50 \%$ prevalence of sleep disturbance in individuals with COPD, the number of studies focusing on whether dyspnoea or other COPD symptoms is the cause of these disturbances is very small $[15,16]$. In a randomised controlled trial of fluticasone propionate/ salmeterol in patients with severe COPD, $\sim 35 \%$ reported night-time awakenings due to COPD [16]. This is higher than in the most severe category in our population and is most probably related to the fact that the patients were recruited into the trial because they were symptomatic, whereas subjects included in our analyses were attending a general health examination.

The individuals with night-time dyspnoea more often reported feelings of stress and tiredness. In a study like ours, it is not possible to conclude whether anxiety and tiredness was a cause or a consequence of nighttime awakening due to dyspnoea, but both explanations are plausible. Sleep disturbances can affect healthrelated quality of life, and stress and anxiety can also result in night time awakenings [17]. Prospective studies have related poor sleep quality and sleep disturbance in COPD to increased subsequent morbidity and mortality $[3,13]$. In keeping with these findings, our study also shows that waking up at night due to dyspnoea is a marker of poor prognosis in COPD. In individuals with this symptom, both exacerbations and COPD admissions were more common during follow-up, and survival was poorer. Even after statistical adjustment for the spirometry-based GOLD 1-4 staging, the excess risk of experiencing an exacerbation or acute admission due to COPD was significantly elevated, whereas after an adjustment for the clinical GOLD A-D stage (which includes daytime dyspnoea as one of the parameters), only the risk of exacerbations was significantly elevated, with a hazard ratio of 1.4 .

The present study has both strengths and weaknesses. The strengths include the prospective design, comprehensive registration of respiratory risk factors and complete follow-up. With regard to diagnosis of COPD admissions based on hospital records, a previous study has identified a high specificity and an 
TABLE 4 The risk of exacerbations, hospital admissions and death among participants with chronic obstructive pulmonary disease according to night-time dyspnoea, derived from a Cox regression model with age as the underlying time scale

Univariate model adjusted for age and sex
Multivariate model adjusted for age, sex and GOLD 1-4"
Multivariate model adjusted for age, sex and GOLD A-D?

\section{Exacerbations ${ }^{+}$ \\ No night-time dyspnoea \\ Night-time dyspnoea \\ Admissions $^{\S}$ \\ No night-time dyspnoea \\ Night-time dyspnoea \\ All-cause mortality ${ }^{f}$ \\ No night-time dyspnoea \\ Night-time dyspnoea}

1 (ref.)

$2.28(1.74-3.00)$

1 (ref.)

$3.18(2.30-4.39)$

1 (ref.)

$1.65(1.19-2.27)$
1 (ref.)

$1.79(1.36-2.36)$

1 (ref.)

$2.07(1.49-2.87)$

1 (ref.)

$1.37(0.99-1.89)$
1 (ref.)

$1.39(1.05-1.84)$

1 (ref.)

$1.30(0.93-1.81)$

1 (ref.)

$1.03(0.74-1.44)$

Data are presented as hazard ratio ( $95 \%$ confidence interval). The hazard ratio is considered statistically significant if the confidence interval does not include 1 (bold). GOLD: Global Initiative for Chronic Obstructive Lung Disease. \#: spirometry-based classification of COPD based on forced expiratory volume in $1 \mathrm{~s}$ (FEV1) as a percentage of the predicted value; " clinical classification according to FEV1 \% pred, symptom burden and frequency of exacerbations; ${ }^{+}: n=687 ;{ }^{\S}: n=359 ;{ }^{f}: n=591$.

adequate completeness of COPD diagnosis in Danish Hospitals [18]. Weaknesses of our study include the fact that the number of individuals with severe COPD was relatively small, as most of the participants in the Copenhagen studies have mild COPD. The fact that we only had pre-bronchodilatory spirometry is also a drawback, but when we performed our analyses on a subgroup with FEV1/FVC < LLN, we found even stronger associations between night-time dyspnoea and prognosis. Thus, we do not think that selection of the individuals on the basis of pre-bronchodilatory spirometric values influences the observed association between night-time dyspnoea and COPD. Finally, the lack of information on night-time respiratory symptoms other than dyspnoea is also a drawback and, therefore, additional studies with a more precise characterisation of night-time disturbances in COPD are needed.

In conclusion, our study of a large sample of individuals with COPD selected from the general population shows that the prevalence of night-time dyspnoea increases with disease severity, and is associated with presence of daytime respiratory symptoms and cardiac comorbidities. Night-time dyspnoea is a marker of poor prognosis with regard to exacerbations, hospital admissions and survival.

\section{References}

1 Global Initiative for Asthma. Global Strategy for Asthma Management and Prevention. www.ginasthma.org/local/ uploads/files/GINA_Report_March13.pdf Date last updated: 2012.

2 Jones P, Harding G, Berry P, et al. Development and first validation of the COPD assessment test. Eur Respir J 2009; 34: 648-654.

3 Augusti A, Hedner J, Marin JM, et al. Night-time symptoms: a forgotten dimension of COPD. Eur Respir Rev 2011; 121: 183-194.

4 Appleyard M. The Copenhagen City Heart Study. Scand J Soc Med, 1989: Suppl. 41, 1-160.

5 Lange P, Mogelvang R, Marott JL, et al. Cardiovascular morbidity in COPD: a study of the general population. COPD 2010; 7: 5-10.

6 Stender S, Frikke-Smidth R, Nordestgaard BG, et al. Sterol transporter adenosine triphosphate-binding cassette transporter G8, gallstones, and biliary cancer in 62,000 individuals from the general population. Hepatology 2011; 53: 640-648.

7 Vestbo J, Hurd SS, Agusti AG, et al. Global strategy for the diagnosis, management, and prevention of chronic obstructive pulmonary disease: GOLD executive summary. Am J Respir Crit Care Med 2013; 187: 347-365.

8 Løkke A, Marott JL, Mortensen J, et al. New Danish reference values for spirometry. Clin Respir J 2013; 7: 153-167.

9 Lange P, Marott JL, Vestbo J, et al. Prediction of the clinical course of chronic obstructive pulmonary disease, using the new GOLD classification. Am J Respir Crit Care Med 2012; 186: 975-981.

10 Thomsen M, Marott JL, Ingebrigtsen TS, et al. Inflammatory biomarkers in chronic obstructive pulmonary disease. JAMA 2013; 309: 2353-2361.

11 Gaist D, Sørensen HT, Hallas J. The Danish prescription registries. Dan Med Bull 1997; 44: 445-448.

12 The Comprehensive R Archive Network. http://cran.r-project.org/ Date last accessed: May 21, 2013. Date last updated: September 25, 2013.

13 Omachi TA, Blanc PD, Claman DM, et al. Disturbed sleep among COPD patients is longitudinally associated with mortality and adverse COPD outcomes. Sleep Med 2012; 13: 476-483.

14 Price D, Small M, Milligan G. The prevalence and impact of nighttime symptoms in COPD patients - results from a cross-sectional study in five European countries. Proceedings of the IV World Asthma and COPD Forum (Paris, France, April 30 to May 3, 2011). 
15 Anzueto A, Ferguson GT, Feldman G, et al. Effect of fluticasone propionate/salmeterol (250/50) on COPD exacerbations and impact on patient outcomes. COPD 2009; 6: 320-329.

16 Tashkin DP, Rennard SI, Martin P, et al. Efficacy and safety of budesonide and formoterol in one pressurized metered-dose inhaler in patients with moderate to very severe chronic obstructive pulmonary disease: results of a 6-month randomized clinical trial. Drugs 2008; 68: 1975-2000.

17 Balkin TJ, Rupp T, Picchioni D, et al. Sleep loss and sleepiness: current issues. Chest 2008; 134: 653-660.

18 Thomsen RW, Lange P, Hellquist B, et al. Validity and underrecording of diagnosis of COPD in the Danish National Patient Registry. Respir Med 2011; 105: 1063-1068. 